\title{
A Simple Modulation Code with Peak Power Reduction and Coding Gain
}

\author{
Makoto Tanahashi and Hideki Ochiai \\ Department of Electrical and Computer Engineering - Yokohama National University \\ Yokohama, Kanagawa 240-8501, Japan \\ Email: makoto@ochiailab.dnj.ynu.ac.jp, hideki@ynu.ac.jp
}

\begin{abstract}
In this paper, a simple modulation code called symbol insertion for band-limited single-carrier systems is proposed. This code can reduce the peak-to-average power ratio (PAR) with an inherent forward error correction (FEC) capability. The idea of the proposed code is as follows; since the PAR is largely proportional to the phase shifts of consecutive discrete symbols, we eliminate large phase shifts by inserting redundant symbols every after information symbols. Furthermore, exploiting the fact that the insertion process has a trellis structure, we employ the symbol insertion as a component code of concatenated codes. An 8-PSK example demonstrates not only that the PAR reduction performance is superior to conventional PAR reduction schemes, but also that the coding gain is as large as those of conventional capacity-approaching turbo and LDPC codes. Another advantage of the proposed system is simplicity of both coding and decoding processes.
\end{abstract}

\section{INTRODUCTION}

Controlling peak-to-average power ratio (PAR) of transmitted signals is a critical issue for band-limited single-carrier transmissions with linear modulations such as phase shift keying (PSK) or quadrature amplitude modulation (QAM). In a band-limited single-carrier PSK, the PAR highly depends on successive phase patterns of discrete complex symbols, and thus the PAR can be reduced by the use of a signal constellation with small maximum phase transition. By limiting the maximum phase transition to 135 [deg], the $\pi / 4$-shift QPSK can achieve PAR reduction of approximately $0.5 \mathrm{~dB}$ with a moderate roll-off factor of pulse-shaping filter. However, it is less effective to apply such an approach to high-order PSK constellations.

One interesting alternative to the constellation-rotating approach is the use of the Ternary-PSK (TPSK or 3-PSK) constellation [1]. The TPSK symbol consists of the three signal points with equilateral triangular geometry. The maximum phase transition is thus restricted to $360 / 3=120$ [deg], which results in a signal with the PAR lower than that of the $\pi / 4$ shift QPSK. The concept of TPSK can be more generally extended to regular $M$-sided polygons where $M$ is an odd number. However, as $M$ increases, this approach becomes less effective in terms of PAR reduction capability, similar to those with constellation rotation.

Since these constellation-oriented techniques have a limited peak power control capability, several sequence-oriented approaches which employ the idea of modulation codes, e.g. [2-4], have been proposed recently. These approaches are effective, but the complexity (especially at the transmitter side) could be prohibitively high for some low-cost applications.

In this paper, as a low-complexity alternative to [2-4], we propose a simple modulation code for the PAR reduction of single-carrier systems. As illustrated in Fig. 1, this scheme controls the phase shifts (and reduces the associated PAR) by inserting a (redundant) controlling symbol after every information symbol. We refer to this scheme as symbol insertion (SI). Due to the insertion of controlling symbols, the maximum allowable phase transition can be reduced to as small as $180 / 2=90$ [deg] in any signal constellation. Hence, the resulting PAR is lower than those of the above mentioned constellation-oriented schemes. Furthermore, the increment in computation is almost negligible since the controlling symbols to be inserted can be determined and tabulated beforehand for given anteroposterior information symbols.

At first glance, the loss associated with the symbol insertion appears prohibitive. However, the inserted symbols can be viewed as parities for information symbols, which thus allows us to exploit them for error correction. The reduced transmission rate can be compensated for in this way. In block diagram notation, the SI can be represented as a convolutional code (CC) as sketched in Fig. 2 (a). The major difference of this $M$-ary $\mathrm{CC}$ from the binary $\mathrm{CC}$ is that it operates with $M$ ary alphabet, instead of binary, for $M$-ary PSK modulation. Due to this trellis structure, the SI can be effectively decoded by Viterbi or BCJR algorithms. Furthermore, the system combined with another (possibly binary) CC (as illustrated in Fig. 3) can be viewed as a special case of serially concatenated turbo codes [5], and thus the iterative (turbo) decoding is applicable. In serial concatenation systems, it is desired that an inner code has a recursive structure to enjoy interleaver gain [6]. Therefore, we also propose Differentially-encoded Symbol Insertion (DSI) shown in Fig. 2 (b). We have confirmed by simulation that it achieves coding gain as large as that of the original turbo codes.

\section{System Model AND Definitions}

Let us denote an $M$-PSK constellation by

$$
\mathcal{S}_{M}=\left\{S^{i}=e^{j 2 \pi i / M} \mid 0 \leq i<M\right\},
$$

where the variable $i$ denotes the constellation index. Let $\boldsymbol{i}=\left\{i_{n} \mid 1 \leq n \leq N\right\}$ be a sequence of the indices of consequent symbols of length $N$, representing information, 


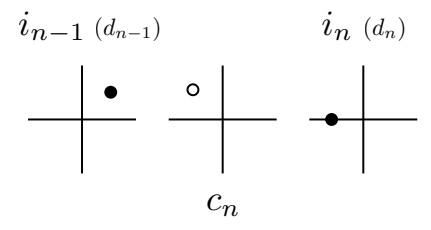

- information symbol (or, differentially encoded symbol)

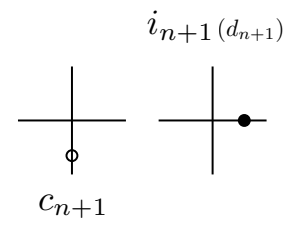

o controlling symbol
Fig. 1. The proposed symbol insertion system. Large phase rotation can be eliminated by the presence of judiciously inserted symbols.
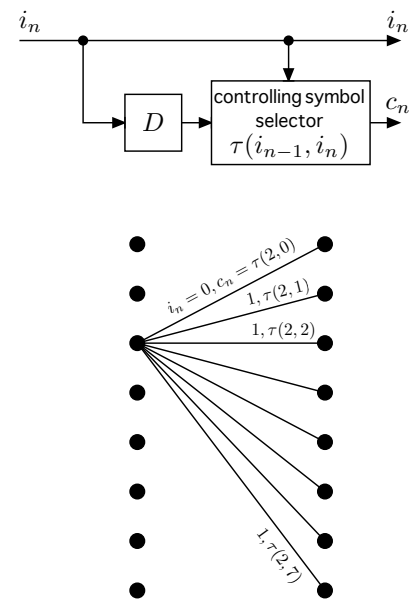

(a)
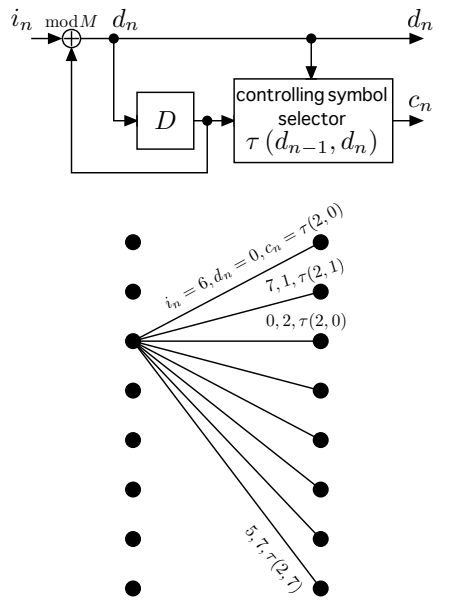

(b)
Fig. 2. Block and trellis diagrams (in the case of $M=8$ ) of symbol insertion systems. (a) Symbol Insertion (SI). (b) Differentially-encoded Symbol Insertion (DSI).

possibly after channel coding. Here, we consider the following two transmission schemes: a) $\boldsymbol{i}$ is directly fed to the channel, or b) $\boldsymbol{i}$ is encoded into $\boldsymbol{d}$ by differential encoder (DE) which is defined as

$$
d_{n}=d_{n-1} \oplus i_{n}
$$

where $d_{0} \triangleq 1$ is a non-data carrying symbol and the operator $\oplus$ denotes modulo- $M$ addition. Similarly, modulo- $M$ subtraction will be denoted by $\ominus$.

For a given information sequence $i$ or its differentiallyencoded counterpart $\boldsymbol{d}$, the controlling symbol selector in Fig. 2 determines a controlling symbol index $c_{n}$ to be inserted between $i_{n-1}$ and $i_{n}$ (or $d_{n-1}$ and $d_{n}$ ). In the following, we explicitly denote this operation by $\tau(\cdot, \cdot)$. In the case without $\mathrm{DE}$, the insertion symbol index is given by

$$
c_{n}=\tau\left(i_{n-1}, i_{n}\right),
$$

and the final form of transmitted baseband symbol vector $\boldsymbol{S}$ is given by $\boldsymbol{S}=\left\{\ldots, S^{c_{n-1}}, S^{i_{n-1}}, S^{c_{n}}, S^{i_{n}}, \ldots\right\}=$ $\left\{\ldots, \boldsymbol{S}_{n-1}, \boldsymbol{S}_{n}, \ldots\right\}$ where $\boldsymbol{S}_{n} \triangleq\left\{S^{c_{n}}, S^{i_{n}}\right\}$. Note that $i_{n}$ is replaced by $d_{n}$ in the case of the DSI. We assume an AWGN channel with the two-sided power spectral density $N_{0} / 2$, and denote the noise vector by $\boldsymbol{W}$. The received symbol vector $\boldsymbol{R}$ is then expressed as $\boldsymbol{R}=\boldsymbol{S}+\boldsymbol{W}$. Since the received values

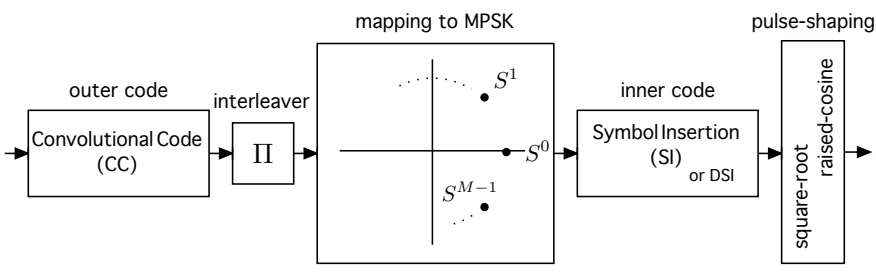

Fig. 3. A transmitter model with $M$-PSK modulation.

are continuous, we denote a pair of received symbols at the $n$th time slot by $\boldsymbol{R}_{n} \triangleq\left\{R_{n}^{(c)}, R_{n}^{(i)}\right\}$, which corresponds to the received version of $\boldsymbol{S}=\left\{S^{c_{n}}, S^{i_{n}}\right\}$.

The transmitter model considered in this paper is depicted in Fig. 3. In this transmitter, the SI (or DSI) carries out the insertion operation with respect to the symbol indices $i$ after convolutional coding (CC) and random interleaving. Hence, it works as an inner component code of a serially concatenated system. As a constellation, Gray labeling is assumed as an example. Also, we adopt a square-root raised-cosine pulse for the pulse-shaping filter.

\section{SYMBOL INSERTION}

\section{A. Basic Rule for Insertion}

We propose a systematic design of the insertion table $\tau(\cdot, \cdot)$. For simplicity of design, we introduce the property of rotational invariance to the function $\tau(\cdot, \cdot)$. To this end, we first define $\tilde{\tau}(i)$ as

$$
\tilde{\tau}(i) \triangleq \tau(0, i)
$$

and assume that $\tau(i, j)$ satisfies

$$
\tau(i, j)=i \oplus \tilde{\tau}(j \ominus i) .
$$

The above property indicates that the phase rotation $\tilde{\tau}(\cdot)$ given by a controlling symbol depends only on the index difference of two adjacent symbols and not their individual values. Due to the introduction of this rule, we need only specify the controlling symbols inserted between the symbol with index 0 and the next symbol with $M$ index patterns. Our objective here is to find a $\tilde{\tau}$ with both low PAR (i.e., low phase shifts) and good error-correcting capability.

\section{B. Constraints for PAR reduction}

From the viewpoint of PAR reduction, we impose a constraint such that the maximum phase shift is less than 90 [deg]. Specially, for a given $i, c \triangleq \tilde{\tau}(i)$ should satisfy,

$$
\left|\arg S^{c}\right|,\left|\arg \frac{S^{c}}{S^{i}}\right| \leq 90[\mathrm{deg}] .
$$

This is equivalent to imposing the following rule.

Rule 1: Minimization of maximum phase transition (MMP)

$$
\left\{\begin{array}{l}
c \leq \frac{M}{4}, M-\frac{M}{4} \leq c, \\
c \ominus i \leq \frac{M}{4}, M-\frac{M}{4} \leq c \ominus i .
\end{array}\right.
$$


TABLE I

EXAMPLES OF INSERTION TABLES $\tilde{\tau}(i)$ FOR 8-PSK. (A) OPTIMAL PATTERNS FOUND IN THE MMP SET UNDER THE DISTANCE DISTRIBUTION CRITERION. (B) EXAMPLES OF BAD PATTERNS IN THE MMP SET. (C) AN OPTIMAL PATTERN FOUND IN THE MMP-ZPE SET.

\begin{tabular}{c|r|cccccccc}
\hline & information symbol $i$ & 0 & 1 & 2 & 3 & 4 & 5 & 6 & 7 \\
\hline \multirow{4}{*}{ (a) } & $\tilde{\tau}_{1}$ & 1 & 7 & 2 & 1 & 2 & 6 & 0 & 6 \\
& $\tilde{\tau}_{2}$ & 2 & 0 & 2 & 1 & 2 & 6 & 0 & 6 \\
& $\tilde{\tau}_{3}$ & 6 & 1 & 0 & 2 & 2 & 7 & 6 & 1 \\
& $* \tilde{\tau}_{4}$ & 6 & 2 & 0 & 2 & 2 & 7 & 6 & 1 \\
& $\tilde{\tau}_{5}$ & 7 & 2 & 0 & 2 & 2 & 7 & 6 & 1 \\
\hline \multirow{2}{*}{ (b) } & $\tilde{\tau}_{\text {ad1 }}$ & 0 & 1 & 2 & 1 & 2 & 7 & 6 & 7 \\
& $\tilde{\tau}_{\text {bad2 }}$ & 0 & 0 & 1 & 1 & 2 & 7 & 6 & 7 \\
\hline (c) & $\tilde{\tau}_{\text {zero }}$ & 6 & 2 & 1 & 1 & 2 & 6 & 7 & 1 \\
\hline \multicolumn{7}{c}{ * optimal in the AMI criterion. }
\end{tabular}

Further PAR reduction can be achieved by eliminating zero phase rotation. Hence, in addition to the MMP rule, we may also impose the following rule:

Rule 2: Zero-phase shift elimination (ZPE)

$$
c \ominus i \neq 0 .
$$

Note that this additional constraint substantially reduces the number of possible candidates for $\tilde{\tau}$, which may thus preclude some of good patterns in terms of error correcting capability found in the MMP candidate set. In the following subsections, we describe our criteria to choose the pattern with the best error-correction capability within the MMP set or the MMPZPE subset.

\section{Distance Distribution Criterion}

One straightforward approach to selecting a good insertion pattern from channel coding perspective is to optimize the distance distribution of the $\mathrm{CC}$ encoder shown in Fig. 2 (a).

The trellis diagram of Fig. 2 (a) shows that every state is connected to every next state by the branch (transition) denoting the observation of one information symbol. Thus, the most likely error events span two-symbol interval, and examining this interval would suffice to obtain the desired distance distribution. The number of possible paths within the two-symbol interval is $M^{3}$ with an $M$-ary alphabet. It should be mentioned that the SI is not a linear code, similar to trellis coded modulation (TCM). Therefore, all the combinations of the two paths among $M^{3}$ paths need to be examined in order to obtain the distance distribution. Let us write the distance distribution by polynomial notation $T(X)=\sum a_{x} X^{x}$ where $x$ denotes the squared sum of Euclidean distances of two paths, $a_{x}$ is the path coefficient representing the number of the paths having the distance $x$, and $X$ is a dummy variable for enumeration.

Design Example with 8-PSK: In the case of 8-PSK, there are 2880 entries in the MMP candidate set. For all the 2880 possible insertion tables, we have computed $T(X)$ and found the best five patterns that have the largest minimum distance $x_{\min }$ and the smallest non-zero coefficient $a_{x_{\min }}$. These insertion tables are listed in Table I. The first three terms of their distance distributions from minimum $x_{\min }$ are as follows.

$$
\begin{aligned}
& T_{\tilde{\tau}_{1}}(X)=16 X^{1.17}+16 X^{1.76}+16 X^{2.00}+\cdots, \\
& T_{\tilde{\tau}_{2}}(X)=16 X^{1.17}+32 X^{1.76}+48 X^{2.00}+\cdots, \\
& T_{\tilde{\tau}_{3}}(X)=16 X^{1.17}+32 X^{1.76}+48 X^{2.00}+\cdots, \\
& T_{\tilde{\tau}_{4}}(X)=16 X^{1.17}+16 X^{1.76}+48 X^{2.00}+\cdots, \\
& T_{\tilde{\tau}_{5}}(X)=16 X^{1.17}+16 X^{1.76}+16 X^{2.00}+\cdots
\end{aligned}
$$

Strictly speaking, the optimal insertion pattern in this criterion is $\tilde{\tau}_{1}$ or $\tilde{\tau}_{5}$, as their second and latter terms indicate. Nevertheless, the first term, which dominates the error event for high SNR, is the same for the above five patterns. They are thus expected to yield no noticeable difference in terms of asymptotic error performance. For comparison, we here present some bad instances:

$$
\begin{aligned}
& T_{\tilde{\tau}_{\text {bad }}}(X)=280 X^{1.17}+72 X^{2.00}+\cdots, \\
& T_{\tilde{\tau}_{\text {bad } 2}}(X)=64 X^{0.59}+192 X^{1.17}+80 X^{1.76}+\cdots .
\end{aligned}
$$

When the ZPE rule is also imposed, we have only 64 entries. The optimal insertion table among these entries has found to be $\tilde{\tau}_{\text {zero }}$ in Table I of which the distance distribution is

$$
T_{\tilde{\tau}_{\text {zero }}}(X)=16 X^{0.59}+64 X^{1.17}+48 X^{1.76}+\cdots .
$$

We can observe that the minimum distance $x_{\min }$ is reduced because of the additional ZPE constraint.

Using the distance distribution polynomial, we can derive a lower bound of the SI with Viterbi decoding as:

$$
P_{\mathrm{SI}, \text { Viterbi }} \geq \frac{2}{\log _{2} M} \sum_{x} \frac{a_{x}}{M^{3}} Q\left(\sqrt{\frac{\log _{2} M}{2} \cdot \frac{x E_{b} / N_{0}}{2}}\right) .
$$

\section{Average Mutual Information Criterion}

In capacity-approaching coding systems that employ iterative decoding of serially concatenated SI and CC, the decoder operates at low $E_{b} / N_{0}$, and the existence of a random interleaver complicates the distance distribution. To deal with this coded case, we propose another design rule, which we refer to as an average mutual information (AMI) criterion. Specifically, we recall the fact that the SI imposes correlation into transmitting symbols as a result of constraining phase shifts, and this in turn reduces achievable information rate, i.e., the AMI. In view of this observation, the best insertion pattern should be the one that has maximum AMI for a given operating SNR (in this context, for relatively low $E_{b} / N_{0}$ ), similar to the performance analysis of modulation codes [7].

The AMI subject to the SI constraint is calculated by

$$
I(\boldsymbol{S} ; \boldsymbol{R})=H(\boldsymbol{R})+H(\boldsymbol{R} \mid \boldsymbol{S}),
$$

where $H(\boldsymbol{X})$ denotes the entropy of a stochastic process $\boldsymbol{X}$. The second term, denoting the entropy of a white noise with variance $N_{0} / 2$, is given by a closed form: $H(\boldsymbol{W})=$ $-\log _{2}\left(\pi e N_{0}\right)$. On the other hand, along with the definition of the entropy, the first term is expressed as

$$
H(\boldsymbol{R})=-\lim _{n \rightarrow \infty} \frac{1}{2 n} E\left[\log _{2} p\left(\boldsymbol{R}_{1}^{n}\right)\right],
$$


where we have introduced the notation $\boldsymbol{R}_{1}^{n} \triangleq\left(\boldsymbol{R}_{1}, \cdots, \boldsymbol{R}_{n}\right)$ for convenience, and $p(\cdot)$ is the $n$-dimensional joint probability density function (pdf) associated with the argument. Since the transmitted symbols generated by the SI have a specific stochastic structure, an effective computational technique developed in [8] can be used for calculation of (19). In the case of the SI with $M$-ary alphabets, we carry out the following procedure.

Let us rewrite $p\left(\boldsymbol{R}_{1}^{n}\right)$ as a joint probability of $\boldsymbol{R}_{1}^{n}$ with the state at the $n$th time instant. (The state is defined as a stored symbol in the delay element of Fig. 2.) Hence,

$$
p\left(\boldsymbol{R}_{1}^{n}\right)=\sum_{j=0}^{M-1} p\left(\boldsymbol{R}_{1}^{n}, i_{n}=j\right) .
$$

Then, $\alpha_{n}(j) \triangleq p\left(\boldsymbol{R}_{1}^{n}, i_{n}=j\right)$ is recursively decomposed as

$$
\begin{aligned}
\alpha_{n}(j)= & \sum_{k=0}^{M-1} p\left(\boldsymbol{R}_{n} \mid i_{n}=j, i_{n-1}=k\right) \\
& \cdot \operatorname{Pr}\left(i_{n}=j \mid i_{n-1}=k\right) \alpha_{n-1}(j) \\
= & \frac{1}{M} \sum_{k=0}^{M-1} \underbrace{p\left(R_{n}^{(i)} \mid S^{j}\right)}_{\text {information symbol }} \underbrace{p\left(R_{n}^{(c)} \mid S^{\tau(k, j)}\right)}_{\text {controlling symbol }} \cdot \alpha_{n-1}(k),
\end{aligned}
$$

where $p(R \mid S)$ is the channel transition pdf of AWGN channel and given by $p(R \mid S)=\frac{1}{\pi N_{0}} \exp \left(-\frac{|R-S|^{2}}{N_{0}}\right)$.

Design Example with 8-PSK: Using the above formulae, we have computed the AMI- $E_{b} / N_{0}$ characteristic for all the 2880 patterns belonging to the MMP set of 8-PSK, and compared them at low $E_{b} / N_{0}$ region. In Fig. 4 , several AMI- $E_{b} / N_{0}$ curves are plotted. The results have shown that a good insertion pattern with the distance distribution criterion tends to also have a large value in terms of the AMI. All the five insertion patterns listed in Table I have the AMI value greater than those of the other patterns examined. However, among them, we have observed that $\tilde{\tau}_{4}$ (one marked with asterisk in Table I) clearly outperforms the others. Thus, $\tilde{\tau}_{4}$ can be considered optimal according to the AMI criterion. In the MMP-ZPE subset, we have found that $\tilde{\tau}_{\text {zero }}$ which is optimal in the distance distribution criterion is also optimal according to the AMI criterion.

\section{NumericAl Results}

In the following simulations, we employ 8-PSK and the insertion tables listed in Table I.

\section{A. Peak-to-Average Power Ratio Reduction}

First, we examine the performance of the proposed SI in terms of PAR reduction capability. Fig. 5 shows the complementary cumulative distribution function (ccdf) of the instantaneous power (normalized by the average power) subject to the SI constraint in the cases of roll-off factor 0.1 and 0.4 . As inferred from the maximum allowable phase rotation, we can observe that the proposed SI achieves dynamic rage lower than those of $\pi / 4$-shift QPSK and TPSK constellations [1].

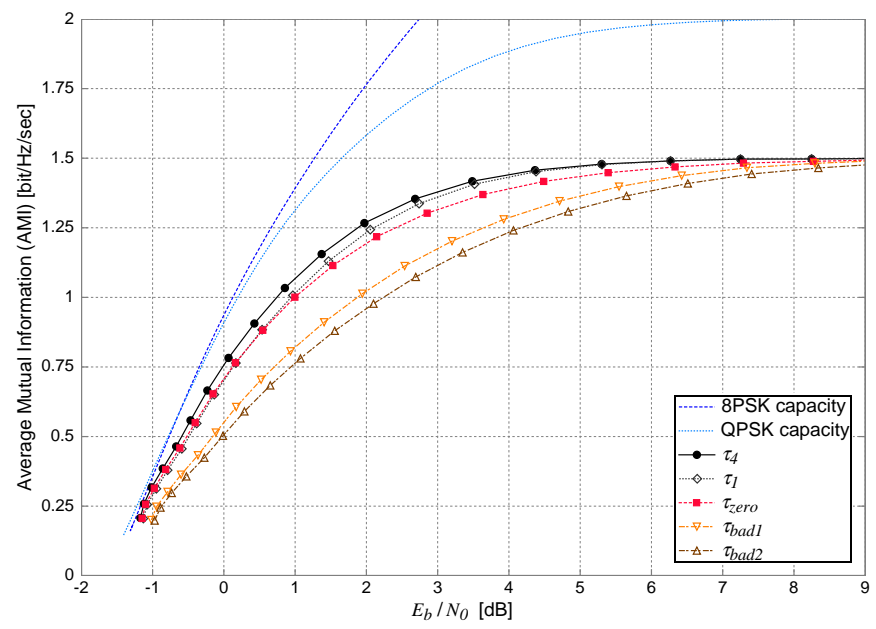

Fig. 4. AMI- $E_{b} / N_{0}$ characteristics of insertion tables. Note that the performance of $\tilde{\tau}_{2}, \tilde{\tau}_{3}$, and $\tilde{\tau}_{5}$ (not shown) is almost identical to that of $\tilde{\tau}_{1}$.

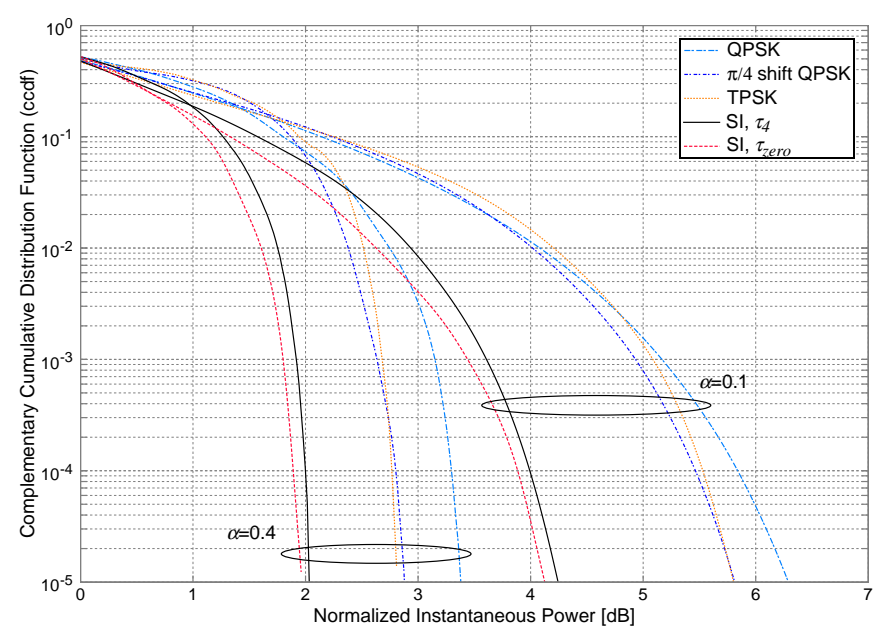

Fig. 5. The ccdf of instantaneous power generated by the 8-PSK modulation with symbol insertion.

We can also observe that eliminating zero-phase difference yields better performance.

\section{B. Bit Error Rate of Viterbi Decoding}

Fig. 6 shows the BER performance when the SI is decoded by Viterbi decoding. As observed, the SI with Viterbi decoding does not outperform the uncoded QPSK, and thus the SI itself cannot achieve coding gain. Note that since the coding process of the SI is systematic, one can decode it by simply extracting the systematic information. For reference, we also plot the performance of this case.

\section{Bit Error Rate of Iterative Decoding}

Next, we examine the BER performance when the SI (DSI) and an outer $\mathrm{CC}$ are concatenated at the transmitter and iteratively decoded at the receiver. In this simulation, the length of the random interleaver is 9000 , and the iteration count is set to 12. The outer CC employed here has a constraint length of $K=3$ and its generator matrix is given by

$$
G_{c}=\left[\begin{array}{ccc}
1 & 0 & \frac{1+D^{2}}{1+D+D^{2}} \\
0 & 1 & \frac{1+D}{1+D+D^{2}}
\end{array}\right] .
$$




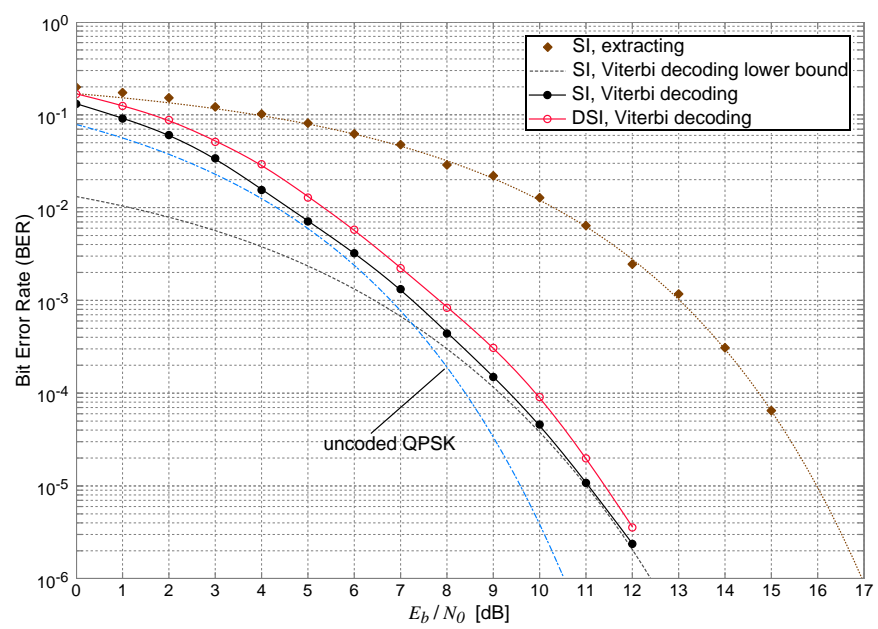

Fig. 6. The BER of the SI and DSI without concatenation and iterative decoding. In this simulation, the optimal insertion table $\tilde{\tau}_{4}$ is employed.

Since the rate of this $\mathrm{CC}$ is $2 / 3$ and that of the $\mathrm{SI}$ is $1 / 2$, the overall rate of the simulated 8 -PSK system is $2 / 3 \cdot \log _{2} 8 \cdot 1 / 2=$ 1 [bit/symbol]. Note that the soft-output decoding process of the proposed concatenation is the same as that given in [5], except that the SI has now an $M$-ary alphabet.

Fig. 7 shows the performance of the SI and DSI along with the vertical lines representing the performance limit derived from the AMI- $E_{b} / N_{0}$ characteristics. As observed, the SI exhibits noticeable error floor, whereas the DSI achieves $\mathrm{BER}=10^{-5}$ within $1.5 \mathrm{~dB}$ of the AMI limit, without error floor. In the figure, the DSIs with $\tilde{\tau}_{\text {zero }}$ and $\tilde{\tau}_{\text {bad2 }}$ are also plotted for comparison. From this result, it is apparent that the design based on the AMI criterion is appropriate for the capacityapproaching system with iterative decoding.

\section{Decoding Complexity}

We quantitatively evaluate the complexity by the number of branch metrics to be calculated for one information bit. In the case of the SI combined with $M$-PSK, one section of the decoding trellis has $M$ states, each connected with $M$ branches. Moreover, since each branch is associated with $\log _{2} M$ information bits, the complexity per information bit is $M^{2} / \log _{2} M$. For $M=8$, the complexity is 21.3 . Likewise, the 2-bit input $\mathrm{CC}$ with $K=3$ given in (22) has the complexity of 4 since there are 4 states, 2 branches per state, and 2-bit input associated with each branch. Consequently, the overall complexity of the serial concatenation simulated here is as small as 25.3 (per iteration).

In existing wireless standards, the turbo codes consisting of the two 1-bit input binary CCs with $K=4$ are widely adopted, e.g., in WiMAX, IMT-2000, and digital video broadcasting (DVB). Based on the same evaluation, we can confirm that the complexity of these standards is 32 (per iteration), which is higher than the proposed serial concatenation. For performance comparison, in Fig. 7, we plot the BER of this turbo code concatenated with QPSK ( $\pi / 4$-shift QPSK). Thereby, the same

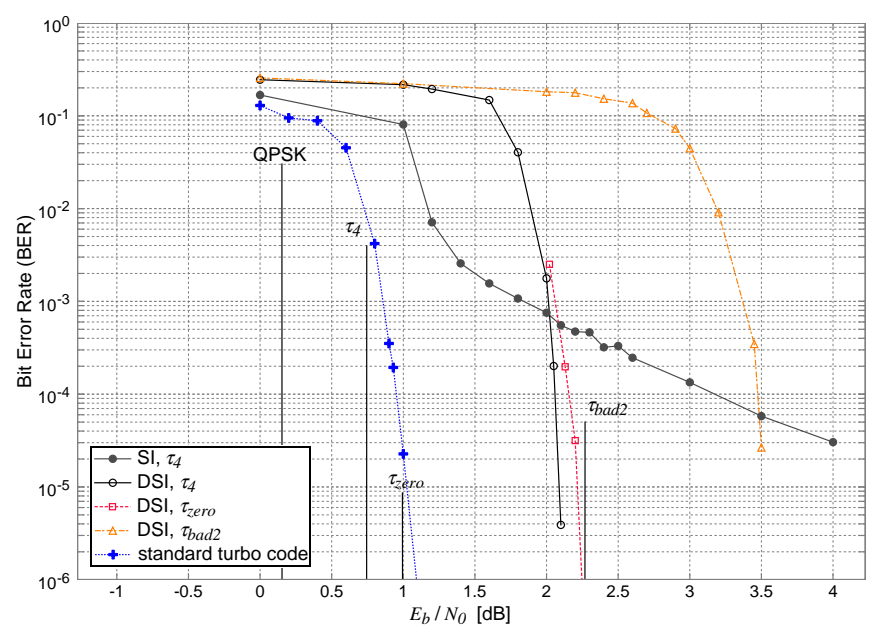

Fig. 7. The BER of the SI and DSI based on iterative decoding with an outer CC.

information rate (1 [bit/symbol]) is achieved by puncturing. We also set the interleaver size and the iteration count equal to those of the serial concatenation of the symbol insertion. As observed, the BER is approximately $1 \mathrm{~dB}$ better than that of the proposed concatenation, but in practice, this little performance degradation may be compensated for by the significant improvement on the PAR.

\section{CONCLUSiON}

In this paper, we have proposed a new modulation code scheme named symbol insertion (SI) for single-carrier systems. It has been demonstrated that the SI offers the significant PAR reduction and coding gain without additional increment in complexity compared to conventional systems.

\section{ACKNOWLEDGMENT}

This work was supported by Industrial Technology Research Grant Program in '05 from New Energy and Industrial Technology Development Organization (NEDO) of Japan.

\section{REFERENCES}

[1] M. Nakamura and H. Torii, "Ternary phase shift keying and its performance," in Proc. IEEE WPMC2002, pp. 1284-1288, Oct. 2002.

[2] M. Chen and O. M. Collins, "Trellis pruning for peak-to-average power ratio reduction,” in Proc. IEEE ISIT'2005, pp. 1261-1265, Sept. 2005.

[3] M. Tanahashi and H. Ochiai, "A new trellis shaping approach for pulseshaped PSK signals with almost constant envelope," in Proc. IEEE ICC'2007, pp. 5598-5603, June 2007.

[4] M. Tanahashi and H. Ochiai, "Near constant envelope trellis shaping for PSK signaling," to appear in IEEE Trans. Commun., 2008.

[5] S. Benedetto, D. Divsalar, G. Montorsi, and F. Pollara, "Serial concatenation of interleaved codes: Performance analysis, design, and iterative decoding," IEEE Trans. Inform. Theory, vol. 44, pp. 909-926, May 1998.

[6] K. R. Narayanan and G. L. Stüber, "A serial concatenation approach to iterative demodulation and decoding," IEEE Trans. Commun., vol. 47, pp. 956-961, July 1999.

[7] J. L. Fan, T. L. Poo, and B. H. Marcus, "Constraint gain," IEEE Trans. Inform. Theory, vol. 50, pp. 1989-2001, Sept. 2004.

[8] D. Arnold and H. A. Loeliger, "On the information rate of binary-input channels with memory," in Proc. IEEE ICC'2001, pp. 2692-2695, June 2001. 